\title{
In-Plane Deformation Mechanics for Highly Stretchable Electronics
}

\author{
Yewang Su, Xuecheng Ping, Ki Jun Yu, Jung Woo Lee, Jonathan A. Fan, Bo Wang, \\ Ming Li, Rui Li, Daniel V. Harburg, YongAn Huang, Cunjiang Yu, Shimin Mao, \\ Jaehoun Shim, Qinglin Yang, Pei-Yin Lee, Agne Armonas, Ki-Joong Choi, Yichen Yang, \\ Ungyu Paik, Tammy Chang, Thomas J. Dawidczyk, Yonggang Huang, Shuodao Wang,* \\ and John A. Rogers*
}

\begin{abstract}
Stretchable electronics represents a relatively recent class of technology ${ }^{[1,2]}$ of interest partly due to its potential for applications in sensory robotic skins, ${ }^{[3,4]}$ conformal photovoltaic modules, ${ }^{[5,6]}$ wearable communication devices, ${ }^{[7,8]}$ skin-mounted monitors of physiological health, ${ }^{[9-11]}$ advanced, soft surgical and clinical diagnostic tools, ${ }^{[11,12]}$ and bioinspired digital cameras. ${ }^{[13,14]}$ A key challenge in each of these systems is in the development of strategies in mechanics that simultaneously allow large levels of elastic stretchability and high areal coverages of active devices built with materials that are themselves not stretchable (e.g., conventional metals) and are, in some cases, highly brittle (e.g., inorganic semiconductors). For design approaches that embed stretchability in interconnect structures that join rigid device islands, the system level stretchability $\varepsilon_{\text {system }}$ is much smaller than the interconnect stretchability $\varepsilon_{\text {interconnect }}$. Zhang et al. ${ }^{[15]}$
\end{abstract}

Prof. Y. Su

State Key Laboratory of Nonlinear Mechanics

Institute of Mechanics

Chinese Academy of Sciences

Beijing 100190, China

Prof. Y. Su, Prof. Y. A. Huang, Prof. Y. Huang

Department of Civil and Environmental Engineering

and Department of Mechanical Engineering

Northwestern University

Evanston, IL 60208, USA

Prof. Y. Su

School of Engineering Science

University of Chinese Academy of Sciences

Beijing 100049, China

Prof. X. Ping

School of Mechanical Engineering

Tianjin University of Science \& Technology

Tianjin 300222, China

Dr. K. J. Yu, Dr. J. W. Lee, Dr. D. V. Harburg, Dr. S. Mao, J. Shim,

Q. Yang, P.-Y. Lee, A. Armonas, K.-J. Choi, Y. Yang, Dr. T. J. Dawidczyk

Frederick Seitz Materials Research Laboratory

Department of Materials Science and Engineering

University of Illinois at Urbana-Champaign

Champaign, IL 61820, USA

Dr. J. W. Lee, U. Paik

Department of Material Science and Engineering

Department of Energy Engineering

Hanyang University

Seoul 133-791, South Korea identified the following relationship between theinterconnectand system stretchability: $\varepsilon_{\text {system }}=\left(1-\sqrt{f_{\text {dev }}}\right) \varepsilon_{\text {interconnect }}$, where $f_{\text {dev }}=$ (area of active device components)/(total area of the system) is the areal coverage ratio of active device components. Structure designs for stretchable interconnects have evolved from straight ${ }^{[13,16]}$ to curvilinear interconnects, ${ }^{[17]}$ from those bonded to or embedded in the supporting substrate ${ }^{[11,18]}$ to free-standing designs housed in microfluidic enclosures, ${ }^{[19]}$ and from simple structures $^{[17]}$ to fractal/self-similar designs. ${ }^{[15,20-22]}$ All such cases, including a broad variety of shapes, sizes, and geometric arrangements, share the same underlying mechanisms, i.e., out-of-plane buckling of thin structures (metals, insulators, or semiconductors with thickness typically between $\approx 100 \mathrm{~nm}$ and $\approx 1 \mu \mathrm{m}$ ) provides the basis for elastic stretchability. The most advanced interconnects achieve elastic (reversible)

DOI: 10.1002/adma.201604989

Prof. J. A. Fan, T. Chang

Department of Electrical Engineering

Stanford University

Stanford, CA 94305, USA

Dr. B. Wang, Prof. S. Wang

School of Mechanical and Aerospace Engineering

Oklahoma State University

Stillwater, OK 74078, USA

E-mail: shuodao.wang@okstate.edu

Dr. M. Li, Dr. R. Li, Prof. Y. Su

State Key Laboratory of Structural Analysis for Industrial Equipment Department of Engineering Mechanics

Dalian University of Technology

Dalian 116024, China

Prof. Y. A. Huang

State Key Lab Digital Manufacturing Equipment and Technology

and Flexible Electronics Research Center

Huazhong University of Science and Technology

Wuhan, Hubei 430074, China

Prof. C. Yu

Department of Mechanical Engineering

University of Houston

Houston, TX 77204, USA

Prof. J. A. Rogers

Department of Electrical and Computer Engineering and the Department of Chemistry Beckman Institute for Advanced Science and Technology

University of Illinois at Urbana-Champaign, Champaign

Urbana, IL 61820, USA

E-mail: jrogers@illinois.edu 
stretchability as high as $\approx 190 \%,{ }^{[15]}$ which corresponds only to $\approx 40 \%$ system stretchability when the areal coverage ratio is $\approx 60 \%$. Lanzara et al. ${ }^{[23,24]}$ introduced a design of networked microwires consisting of a thick layer of kapton film coated with a very thin layer of metal. These microwires can unfold in-plane, with linear response for stretching as large as $750 \%$, and extendable for strains up to $1600 \%$. The elastic stretchability (defined here as the largest cyclic stretching that does not cause irreversible deformation or plastic strain) was, however, not explored in this work. ${ }^{[2]}$ An additional limitation is that interconnects/antennas thin geometries have non-negligible electrical resistances and high levels of power dissipation.

This paper introduces a different route to stretchable structures in which thick bar geometries replace thin ribbon layouts to yield scissor-like deformations instead of in- or out-of-plane buckling modes. Experiments and analytic/computational models show that metal and silicon structures with this bar type configuration can stretch to strains as large as $350 \%$ and $90 \%$, respectively, without fracture. This is a significant improvement over previous studies that achieved $\approx 54 \%$ stretchability for pure-bending, narrow metal interconnects. ${ }^{[25]}$ Metal features with these layouts have the additional advantage that they offer low resistance as interconnects for enhanced operation in examples that include arrays of high-power LEDs and solar cells. In radio frequency (RF) antenna platforms, thick metal reduces RF losses and improves performance.

Experimental (see details in the Experimental Section) and finite element analysis (FEA) studies of the underlying mechanics focus on serpentine interconnects (see Figure 1a for the detailed layouts) made of copper, silicon, and polyimide (PI), with thickness/width aspect ratios between $\approx 1 / 1000$ to $\approx 2.0$. We used thicknesses between $50 \mathrm{~nm}$ and $80 \mu \mathrm{m}$ for a fixed width of $45 \mu \mathrm{m}$ here to focus the discussions. Studies shown in Figure S1 (Supporting Information) on interconnects with other widths show similar dependence on the thickness/width ratios. These interconnects feature very low electrical resistance $(\approx 0.4 \Omega)$, and the changes in resistance with stretching for all types of interconnects are less than a few percent up to the fracture points. In most applications, changes in this range have little practical importance. Investigations begin with copper interconnect traces fabricated on a thin layer PI and then transferred to an elastomer substrate (Ecoflex) as shown in Figure 1a,b. Systematic studies establish the following important aspects of this system:

(1) The existence of three distinct deformation modes: (a) wrinkling for very thin interconnects (sub-micrometer), as reported in prior studies ${ }^{[26,27]}$ and also shown in Figure 1c for $300 \mathrm{~nm}$ and $1 \mu \mathrm{m}$; in this study, wrinkling is defined as out-of-plane buckling without significant twisting, with wavelength smaller than the length of the shortest straight segment (L/2 in Figure 1a); (b) coupled out-of-plane buckling and twisting with relatively long wavelengths $(>L / 2)$ for interconnects with moderate thickness (up to $\approx 45 \mu \mathrm{m}$ ), which we refer to as buckling and is shown in Figure 1c for $12.5 \mu \mathrm{m}$; similar transverse buckling behavior was observed in the study of Yang et al.;[28] (c) pure in-plane bending for even thicker interconnects $(>\approx 45 \mu \mathrm{m})$, which we call scissoring (or non-buckling in comparison to previous studies on buckling interconnects) as shown in Figure 1c for $80 \mu \mathrm{m}$. Detailed deformation of the three modes (amplitudes, wavelengths, and twist angles) appear in Figure S2 (Supporting Information).

(2) A distinct dependence of the elastic stretchability of the interconnects on thickness for a fixed width of $45 \mu \mathrm{m}$. The elastic stretchability corresponds to the largest cyclic stretching level that does not lead to microcracking in the interconnects. In FEA, elastic stretchability is defined as the largest stretching level that does not cause yielding in the interconnects. The cyclic testing and FEA results are shown in Figure 1b. In the wrinkling regime $(<\approx 2 \mu \mathrm{m})$, the elastic stretchability increases and then decreases with increasing thickness, with a maximum of $\approx 40 \%$ at a thickness of $200 \mathrm{~nm} \cdot{ }^{[29]}$ In the buckling regime $(2-50 \mu \mathrm{m})$, the elastic stretchability increases monotonically with the thickness, and then saturates at $\approx 100 \%$ when the thickness reaches $\approx 45 \mu \mathrm{m}$. The stretchability is independent of thickness in the scissoring regime $(>45 \mu \mathrm{m})$.

Transition from Wrinkling to Buckling: The mechanics of wrinkling of a thin metal film on an elastomer substrate gives ${ }^{[2,26,27]}$ the wavelength at the onset of wrinkling as

$$
\lambda \approx 2 \pi\left(\frac{4 S_{\mathrm{int}}}{\bar{E}_{\mathrm{sub}}}\right)^{1 / 3}
$$

where $\bar{E}_{\text {sub }}$ is the plane-strain modulus of the substrate, and

$S_{\text {int }}=\frac{\left(\bar{E}_{\mathrm{PI}} t_{\mathrm{PI}}^{2}-\bar{E}_{\mathrm{m}} t_{\mathrm{m}}^{2}\right)^{2}+4 \bar{E}_{\mathrm{PI}} t_{\mathrm{PI}} \bar{E}_{\mathrm{m}} t_{\mathrm{m}}\left(t_{\mathrm{PI}}+t_{\mathrm{m}}\right)^{2}}{12\left(\bar{E}_{\mathrm{PI}} t_{\mathrm{PI}}+\bar{E}_{\mathrm{m}} t_{\mathrm{m}}\right)}$

is the effective bending stiffness (per unit width) of the interconnect, with $t_{\mathrm{m}}$ and $\bar{E}_{\mathrm{m}}$ being thickness and plane-strain modulus of metal and $t_{\mathrm{PI}}$ and $\bar{E}_{\mathrm{PI}}$ for PI, respectively. (See the Supporting Information for details.) A simple criterion for wrinkling is that the shortest straight segment of length $L / 2$ (see Figure 1a) in the interconnect includes at least one wavelength, i.e.

$$
\lambda<L / 2
$$

Equations (1)-(3) yield a critical metal thickness (see the Supporting Information for details):

$$
t_{\mathrm{cr}}^{\text {wrinkle-buckle }} \approx\left(\frac{3 \bar{E}_{\mathrm{sub}}}{\bar{E}_{\mathrm{m}}}\right)^{1 / 3} \frac{L}{4 \pi}
$$

under the assumptions of $t_{\mathrm{PI}} \sim t_{\text {metal }}$ and $\bar{E}_{\mathrm{PI}}<<\bar{E}_{\text {metal }}$. Equation (4) separates wrinkling $\left(t_{\mathrm{m}}<t_{\mathrm{cr}}^{\text {wrinkle-buckle }}\right)$ from buckling $\left(t_{\mathrm{m}}>t_{\mathrm{cr}}^{\text {wrinkle-buckle }}\right)$. For Ecoflex with $\bar{E}_{\mathrm{sub}}=49.3 \mathrm{kPa}$, copper with $\bar{E}_{\mathrm{m}}=140 \mathrm{GPa},{ }^{[22]}$ and $L=3 \mathrm{~mm}$ in experiments, Equation (4) gives $t_{\mathrm{cr}}^{\text {wrinkle-buckle }}=2.43 \mu \mathrm{m}$, which agrees well with experiments and FEA (Figure 1b,c and Figure S3, Supporting Information).

Transition from Buckling to Scissoring: Although the coupled bending/twisting instability of straight thin-wall bars has been well studied, ${ }^{[30]}$ the scissoring structure here has both straight 
a

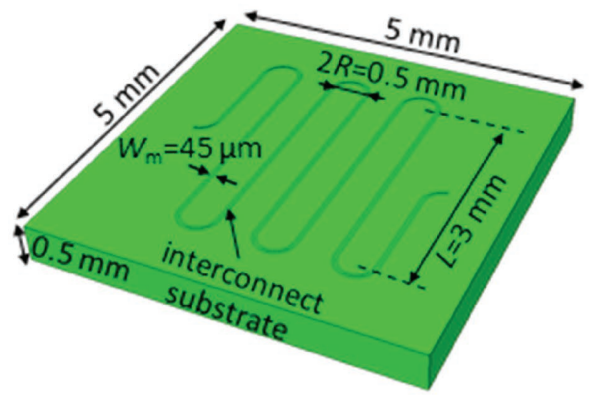

b

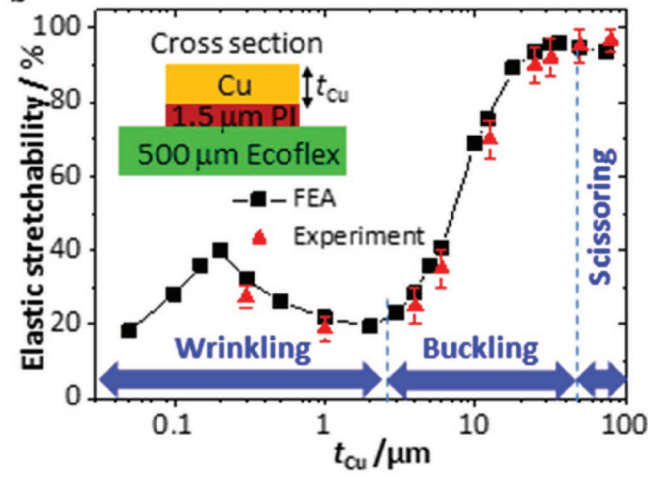

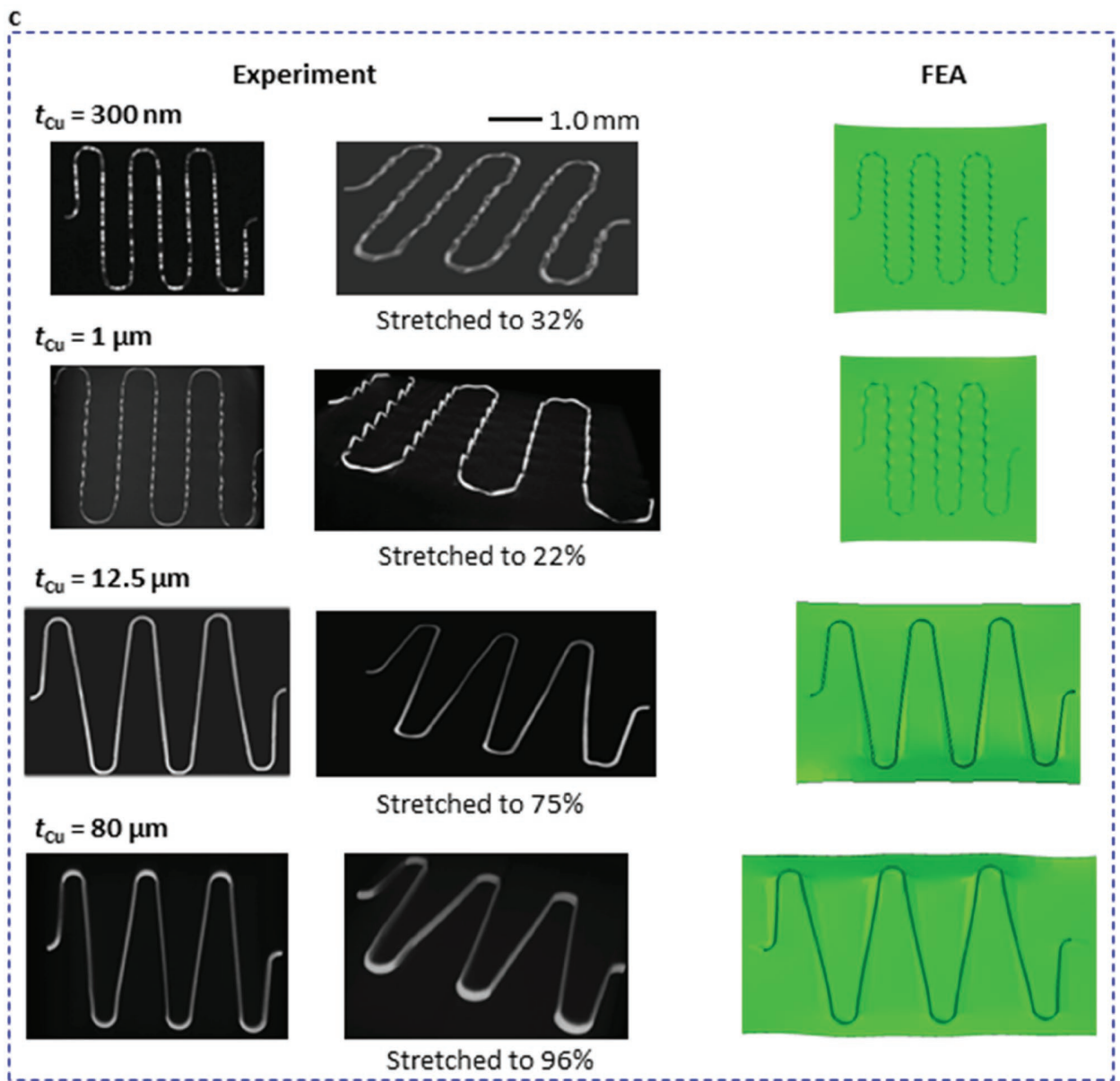

Figure 1. Deformation and elastic stretchability of serpentine interconnects with various thicknesses. a) Layouts of the serpentine interconnects bonded to silicone substrates. b) Experimental fatigue measurement and FEA study of the dependence of the elastic stretchability on the thickness of $\mathrm{Cu}$ interconnects, from $50 \mathrm{~nm}$ to $80 \mu \mathrm{m}$. Three regimes of deformation occur in this range: wrinkling, buckling, and scissoring. c) micro-XCT images and FEA results associated with these regimes, illustrated at thicknesses of $300 \mathrm{~nm}, 1 \mu \mathrm{m}, 12.5 \mu \mathrm{m}$, and $80 \mu \mathrm{m}$, each stretched to the corresponding limit of elastic stretchability.

and curved segments and therefore the buckling mechanism is inherently different. An analytical model is established to analyze the buckling of structures in this study which involves both bending and twisting, characterized by an out-of-plane displacement $v$ (Figure $2 \mathrm{~b}$ ) and torsion angle $\phi$ (Figure 2c), respectively. For a representative unit cell (1) (Figure 2a,b,d) with a straight segment of length $L / 2$ and a quarter circle of radius $R$ stretched by a force $P$, as shown in Figure 2a, results from FEA (Figure 2d, and Figure S4, Supporting Information) suggest that $v$ in Cell 1 can be well described by the kinematically admissible function

$$
v=\left\{\begin{array}{cl}
A_{0} \sin \frac{\pi x}{L}, & 0 \leq x \leq \frac{L}{2} \\
A_{0} \cos \theta, & 0 \leq \theta \leq \frac{\pi}{2}
\end{array}\right.
$$



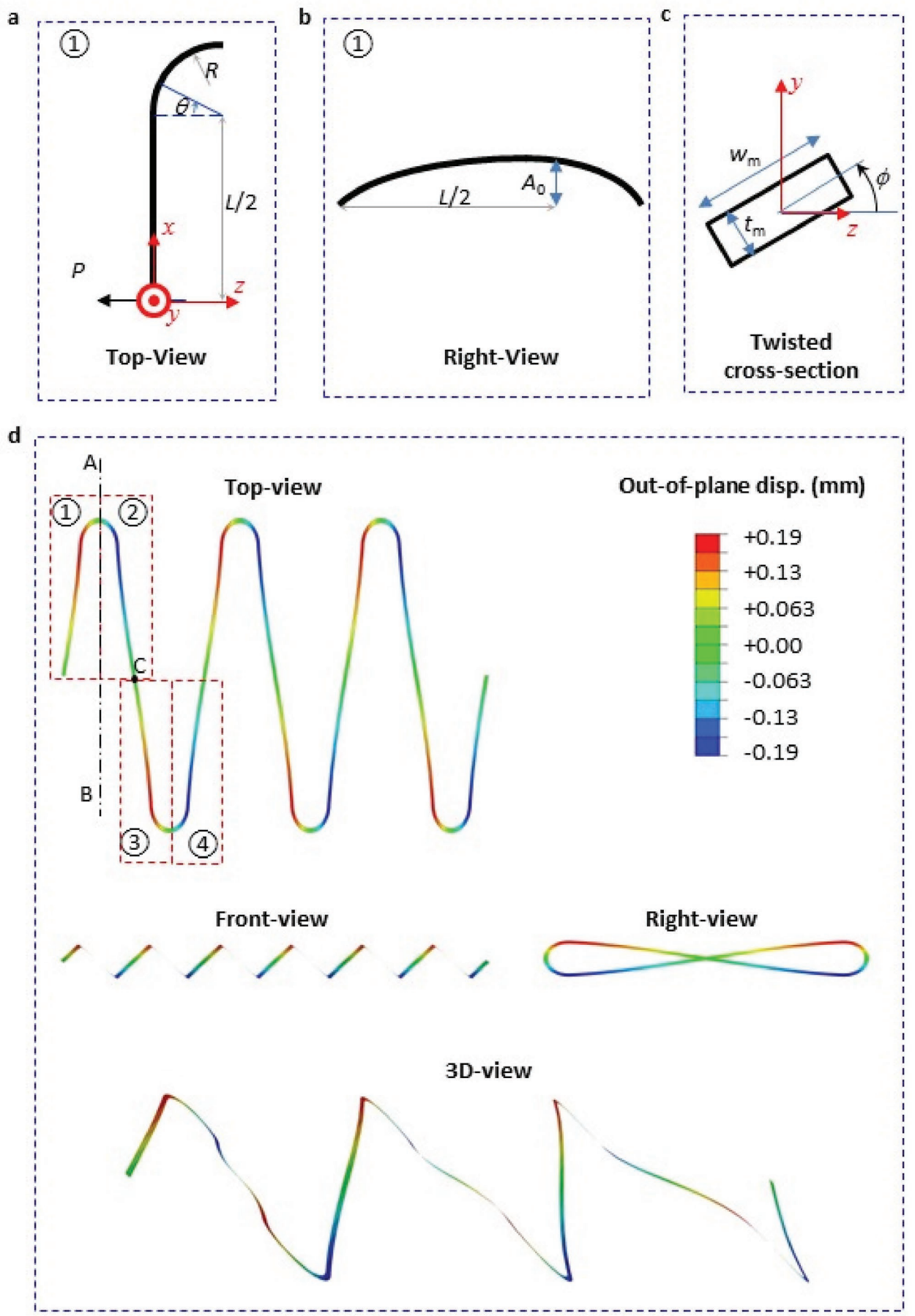

Figure 2. Deformation mode in the buckling regime. a-c) Top-view, right-view, and cross-section view of Cell (1); d) deformation of an interconnect with thickness of $10 \mu \mathrm{m}$, viewed from the top, front, right, and at an angle.

where the coordinates $x$ and $\theta$ are defined in Figure 2a, and the buckling amplitude $A_{0}$ is to be determined (Figure $2 \mathrm{~b}$ ). A periodic structure consists of four cells: Rotating cell (1) by $180^{\circ}$ around Axis AB (Figure 2d) gives cell (2); mirroring cells (1) and (2) about point $\mathrm{C}$ gives cells (3) and (4). Equilibrium gives the torque as $T=-P \nu=G J \cdot \partial \phi / \partial x$ in the straight segment and $T=-P v \cos \theta=(G J / R) \cdot \partial \phi / \partial \theta$ in the curved segment, where GJ is the torsion stiffness. (Here the rotation $\partial v / \partial x$ is proportional to $A_{0} / L$ and is approximated as zero for initial buckling.) This buckling mode has been verified by FEA (shown in Figure 2d, and detailed boundary conditions are discussed in the Supporting Information). Its integration, together with $\left.\phi\right|_{\theta=\pi / 2}=0$ (verified in the FEA results in Figure $2 d$ which shows $\phi$ reaches maximum at $\theta=0$ and vanishes at $\theta=\pi / 2$ ), gives the analytic solution for $\phi$ (see the Supporting Information for details). Let $w_{\mathrm{m}}$ and $E_{\mathrm{m}}$ denote the width of the serpentine interconnect and 
the Young's modulus of the metal, respectively, and $\alpha=R / L$. For an interconnect thickness of $\approx 10 \mu \mathrm{m}$, the effects of the soft substrate are negligible (see the Supporting Information and Figure S5). The energy method is used in the buckling analysis as the following: the potential energy $\Pi_{\text {buckling, }}$ which is the sum of the torsional energy and energy for bending along the width and thickness directions subtracted by the work done by the external load $P$, is obtained in the Supporting Information as

$$
\begin{aligned}
\Pi_{\text {buckling }} & =\Pi_{\text {scissoring }}-\frac{P^{2} A_{0}^{2}}{2 G J} \frac{L}{4}-\frac{P^{2} A_{0}^{2} R}{2 G J} \frac{3 \pi}{16} \\
& -\left(\frac{1}{E I_{2}}-\frac{1}{E I_{1}}\right) \frac{3 P^{4} A_{0}^{2} L^{5}}{2(G J)^{2}} g_{5}(\alpha) \\
& +\left(\frac{1}{E I_{2}}-\frac{1}{E I_{1}}\right) \frac{5 P^{6} A_{0}^{4} L^{7}}{96(G J)^{4}} g_{7}(\alpha)
\end{aligned}
$$

where

$$
\Pi_{\text {scissoring }}=-\frac{P^{2} L^{3}}{48 E I_{1}}\left(1+12 \pi \alpha+48 \alpha^{2}+6 \pi \alpha^{3}\right)
$$

is the potential energy for the scissoring mode (see the Supporting Information for details), $E I_{1}=E_{\mathrm{m}} t_{\mathrm{m}} w_{\mathrm{m}}^{3} / 12$ and $E I_{2}=E_{\mathrm{m}} w_{\mathrm{m}} t_{\mathrm{m}}^{3} / 12$ are the bending stiffness along the width and thickness directions, respectively, and $g_{5}>0$ and $g_{7}>0$ are fifth- and seventh-order polynomials of $\alpha$ (see the Supporting Information for details). Minimization of $\Pi_{\text {buckling }}$ with respect to $A_{0}$, namely $\partial \Pi_{\text {bucklling }} / \partial A_{0}=0$, gives the following equation for $A_{0}$

$A_{0} \cdot\left[\frac{L}{4}+\frac{3 \pi R}{16}+\left(\frac{1}{E I_{2}}-\frac{1}{E I_{1}}\right) \frac{3 P^{2} L^{5}}{G J} g_{5}(\alpha)-\left(\frac{1}{E I_{2}}-\frac{1}{E I_{1}}\right) \frac{5 P^{4} A_{0}^{2} L^{7}}{24(G J)^{3}} g_{7}(\alpha)\right]=0$

It should be noted here that Equation (8) only applies when $P$ is greater than a certain critical buckling force $P_{\mathrm{cr}}>0$. The potential energies are then compared in the Supporting Information (Equations S14-S18). The buckling mode occurs only when it has lower energy than the scissoring mode, i.e., $\Pi_{\text {buckling }}<\Pi_{\text {scissoring. }}$ The result yields a criterion to separate buckling from scissoring as

$t_{\mathrm{cr}}^{\text {buckling-scissoring }}=w_{\mathrm{m}}$

such that buckling and scissoring happen for $t_{\mathrm{m}}<w_{\mathrm{m}}$ and $t_{\mathrm{m}} \geq w_{\mathrm{m}}$, respectively (see the Supporting Information). This simple criterion also agrees well with FEA and experiments (Figure 1b).

In summary, the above analyses yields the following criteria for the three modes (also see a deformation map in Figure S6, Supporting Information)

$$
\begin{aligned}
& \text { wrinkling if } \quad \frac{t_{\mathrm{m}}}{w_{\mathrm{m}}}<\left(\frac{3 \bar{E}_{\mathrm{sub}}}{\bar{E}_{\mathrm{m}}}\right)^{1 / 3} \frac{L}{4 \pi w_{\mathrm{m}}} \\
& \text { buckling if } \quad\left(\frac{3 \bar{E}_{\mathrm{sub}}}{\bar{E}_{\mathrm{m}}}\right)^{1 / 3} \frac{L}{4 \pi w_{\mathrm{m}}}<\frac{t_{\mathrm{m}}}{w_{\mathrm{m}}}<1 \\
& \text { scissoring if } \quad 1<\frac{t_{\mathrm{m}}}{w_{\mathrm{m}}}
\end{aligned}
$$

Stretchability in the Wrinkling Mode: The maximum strain in the metal layer results mainly from out-of-plane bending during wrinkling, and is given by $\varepsilon_{\max }=\kappa_{\max } d$, where the maximum curvature $\kappa_{\max }$ depends on the applied strain (see the Supporting Information for details), and

$d=\frac{\bar{E}_{\mathrm{PI}} t_{\mathrm{PI}}^{2}+2 \bar{E}_{\mathrm{PI}} t_{\mathrm{PI}} t_{\mathrm{m}}+\bar{E}_{\mathrm{m}} t_{\mathrm{m}}^{2}}{2\left(\bar{E}_{\mathrm{PI}} t_{\mathrm{PI}}+\bar{E}_{\mathrm{m}} t_{\mathrm{m}}\right)}$

is the distance from the neutral mechanical plane to the top surface of the metal layer. The stretchability $\varepsilon_{\text {stretchability }}$ is the applied strain when $\varepsilon_{\max }$ reaches the yield strain $\varepsilon_{\text {yield }}$ of the metal, and is determined from (see the Supporting Information for details)

$$
\begin{aligned}
\left(1+\frac{3}{4} \varepsilon_{\text {stretchability }}\right) \sqrt{2 \varepsilon_{\text {stretchability }}} \approx \\
4 \varepsilon_{\text {yield }} \frac{\left(4 S_{\text {int }} / \bar{E}_{\text {sub }}\right)^{1 / 3}\left(\bar{E}_{\mathrm{PI}} t_{\mathrm{PI}}+\bar{E}_{\mathrm{m}} t_{\mathrm{m}}\right)}{\bar{E}_{\mathrm{PI}} t_{\mathrm{PI}}^{2}+2 \bar{E}_{\mathrm{PI}} t_{\mathrm{PI}} t_{\mathrm{m}}+\bar{E}_{\mathrm{m}} t_{\mathrm{m}}^{2}}
\end{aligned}
$$

where $S_{\text {int }}$ is the effective bending stiffness given in Equation (1). The maximum stretchability is reached when the right hand side of Equation (12) attains its maximum, i.e., $\partial() / \partial t_{\mathrm{m}}=0$, which gives the optimal thickness $t_{\mathrm{m}}^{\text {optimum }}$ of the metal as

$$
\frac{t_{\mathrm{m}}^{\text {optimum }}}{t_{\mathrm{PI}}}=f\left(\frac{\bar{E}_{\mathrm{PI}}}{\bar{E}_{\mathrm{m}}}\right)
$$

where the function $f$ is given in the Supporting Information. For $t_{\mathrm{m}}<t_{\mathrm{m}}^{\mathrm{optimum}}$, the stretchability increases with the metal thickness, and the opposite holds for $t_{\mathrm{m}}>t_{\mathrm{m}}^{\mathrm{optimum}}$, which is consistent with Figure $1 \mathrm{~b}$. For $\bar{E}_{\mathrm{PI}}=2.83 \mathrm{GPa}$ and $t_{\mathrm{PI}}=1.5 \mu \mathrm{m}$ for PI, $\bar{E}_{\mathrm{m}}=140 \mathrm{GPa}$ for copper, Equation (13) gives $t_{\mathrm{m}}^{\text {optimum }}=213 \mathrm{~nm}$, which agrees well with $\approx 200 \mathrm{~nm}$ obtained from FEA, as well as experimental results in Figure $1 \mathrm{~b}$.

Stretchability in the Buckling Mode: the experimental and FEA results in Figure 1b show that the stretchability of the buckling mode is a transition from low stretchability of the wrinkling mode to high stretchability of the scissoring mode. Compared to interconnects in the wrinkling and scissoring modes, buckling interconnects are much less attractive options for stretchable electronics. Buckling interconnects are much thicker than wrinkling interconnects and therefore significantly decrease the overall flexibility of the system; on the other hand, they do not take full advantage of the scissoring mechanism to achieve optimum stretchability; out-of-plane twisting associated with the buckling mode also makes it undesirable for most practical designs. Therefore, we only focus on theoretical studies of the stretchability in the wrinkling and scissoring modes.

Stretchability in the Scissoring Mode: Only pure in-plane bending occurs in the scissoring mode. The elastic stretchability is the applied strain when the maximum strain, obtained analytically in the Supporting Information, reaches the yield strain $\varepsilon_{\text {yield }}$ of the metal, which gives

$$
\varepsilon_{\text {stretchability }}^{\text {scissoring }}=\varepsilon_{\text {yield }} \frac{1+12 \pi \alpha+48 \alpha^{2}+6 \pi \alpha^{3}}{2 \alpha\left[\frac{w_{\mathrm{m}}^{2}}{L^{2}}+\frac{3 w_{\mathrm{m}}(1+2 \alpha)}{L}\right]}
$$


where $\alpha=R / L$. This value is independent of the thickness and moduli of metal and substrate, which is confirmed by FEA as shown in Figure S7 (Supporting Information). For $w_{\mathrm{m}} / L<<1$ (e.g., $w_{\mathrm{m}} \approx 0.01 \mathrm{~L}$ in experiments), the elastic stretchability is linearly proportional to $\varepsilon_{\text {yield }} L / w_{\mathrm{m}}$; it increases as the length $L$ of the straight segment increases or the width $w_{\mathrm{m}}$ deceases. This behavior occurs because, for the same in-plane rotation, a longer straight segment gives larger in-plane displacements to accommodate the stretching, while a smaller width $w_{\mathrm{m}}$ reduces the in-plane bending strain which is linearly proportional to $w_{\mathrm{m}} / 2$. For $\varepsilon_{\text {yield }}=0.3 \%,{ }^{[31]}$ the elastic stretchability is shown in Figure 3 a for various $L$ and $R$, which agrees well with FEA for interconnect layouts and sizes identical to those in Figure 1a. The elastic stretchability, normalized by $\varepsilon_{\text {yield }} L / w_{\mathrm{m}}$, is shown versus $\alpha=R / L$ in Figure $3 \mathrm{~b}$, which has a minimum at $\alpha=0.60$. For $\alpha<0.60$ as in all designs of interconnects presented here, the elastic stretchability increases as the radius $R$ (or $\alpha$ ) decreases. When $L$ becomes very small or even approaches zero such as in S-shape interconnects, Equation (14) reduces to $\varepsilon_{\text {stretchability }}^{\text {s-shape }}=\left(3 \pi \varepsilon_{\text {yield }} R^{2}\right) / w_{\mathrm{m}}^{2}$. This realm of geometries falls outside of the scope of present studies but might be interesting to explore in future investigations. Fabrication of serpentine interconnects is most straightforward when the radius is at least twice the interconnect width $w_{m} \cdot{ }^{[32]}$ Therefore, the minimum radius $R=2 w_{\mathrm{m}}$ is used for LED and solar cell array structures to achieve largest stretchability, and Equation (14) then simplifies to $\varepsilon_{\text {stretchability }}^{\text {scissoring }}=\varepsilon_{\text {yield }} L^{2} /\left(12 w_{\mathrm{m}}^{2}\right)$.

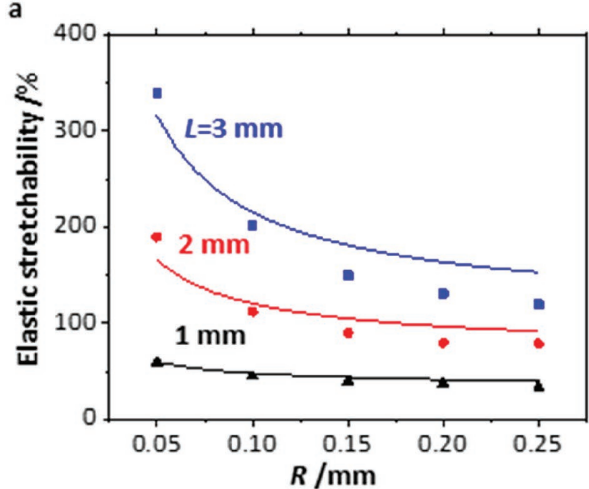

b
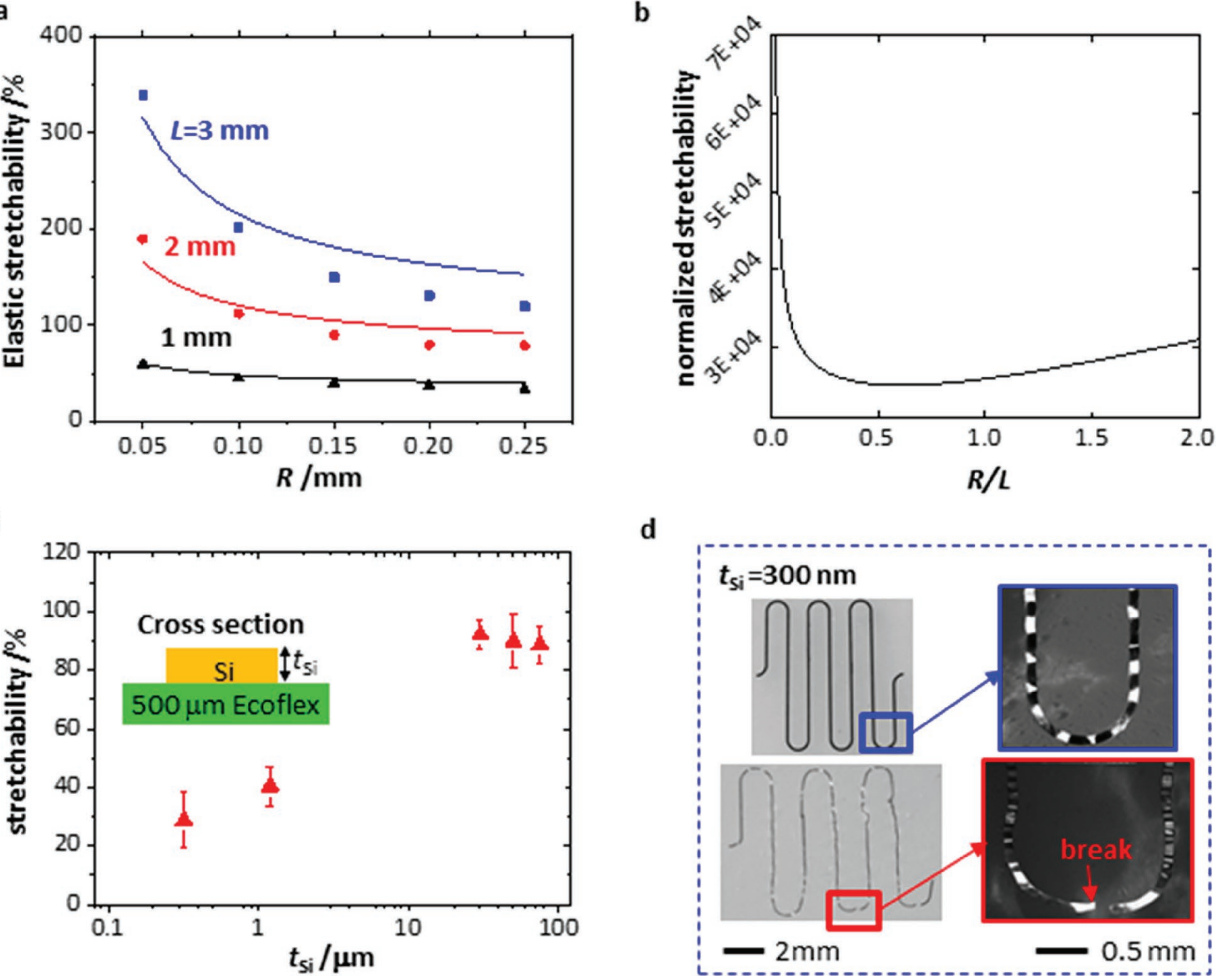

d
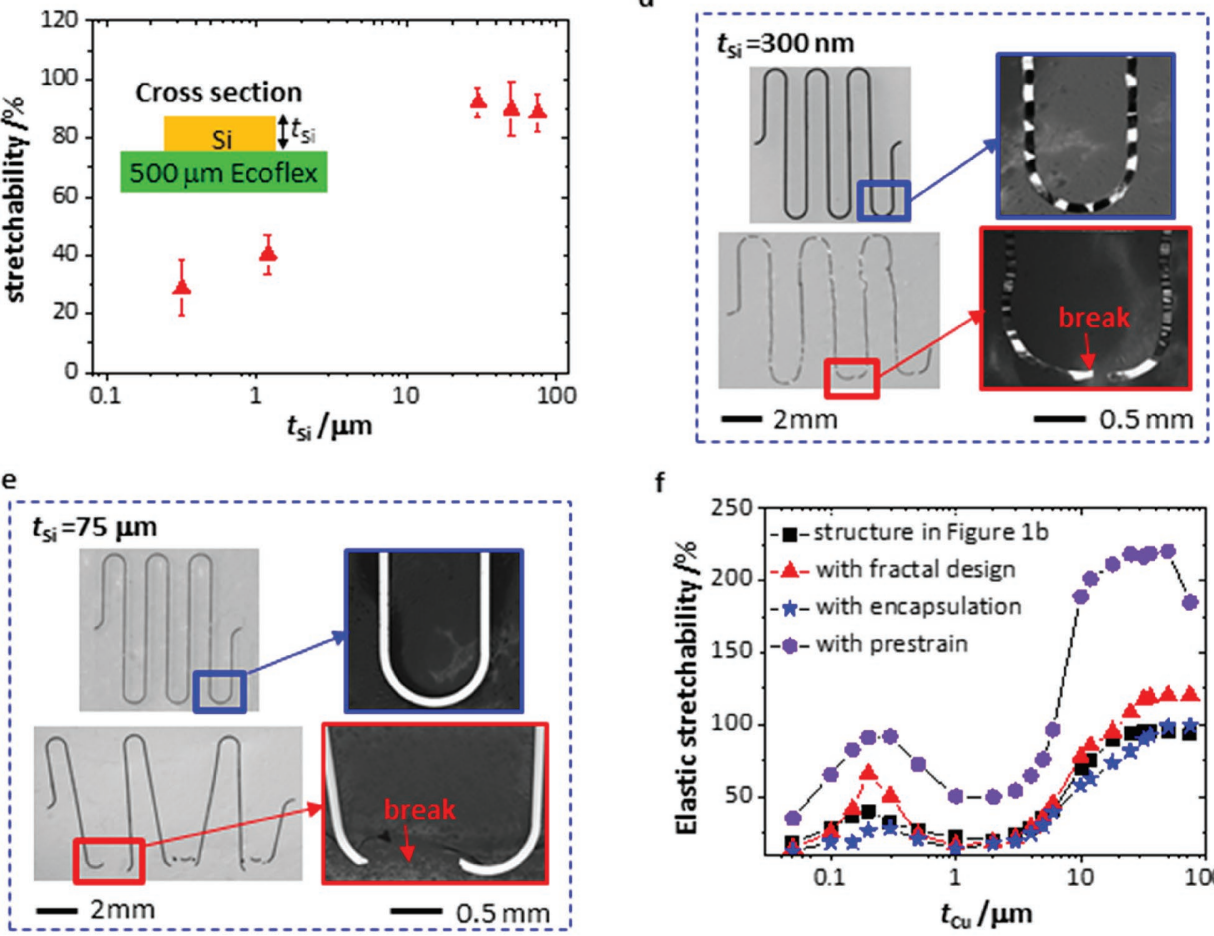

$\mathbf{f}$

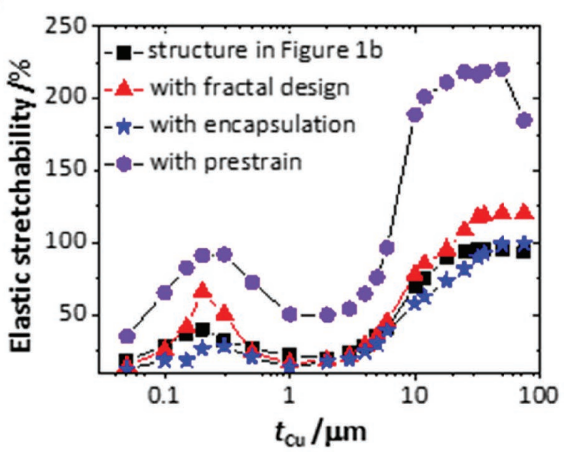

Figure 3. a) Scaling law for the elastic stretchability of interconnects in the scissoring deformation mode. Analytical (solid lines) and FEA (dots) studies of the elastic stretchability of serpentine interconnects with various length $L$ and radius $R$. b) Normalized elastic stretchability as a function of $R / L$. C) Experimental measurement of the stretchability of serpentine structures with silicon thickness ranging from $50 \mathrm{~nm}$ to $100 \mu \mathrm{m}$. C-d) Optical microscopy images of original (top frames) and fractured (bottom frames) serpentines with thicknesses of $300 \mathrm{~nm}$ (d) and $75 \mu \mathrm{m}$ (e). f) The scissoring concept applied in encapsulation, prestrain, and fractal designs. 
The scissoring concept can also be used to enhance the stretchability of brittle inorganic semiconductor materials such as silicon, as shown in Figure 3c-e. Here, stretchability of silicon was measured as the level of stretching (single cycle) that causes fracture in the interconnects. Both experiments (Figure 3c) and FEA (Figure S8 (Supporting Information) which shows the overall trend of stretchability vs thickness) confirm that the scissoring mode enhances the stretchabilitythin ribbons that respond to applied force by wrinkling fracture at an applied strain of $\approx 30 \%$ (Figure $3 \mathrm{~d}$, bottom frames), while thick bars involve scissoring and break at a much higher applied strain of $\approx 90 \%$ (Figure $3 \mathrm{e}$, bottom frames). Scissoring can also be exploited with previously demonstrated approaches in stretchable electronics such as dielectric encapsulation, fractal designs, ${ }^{[20,21,26]}$ and prestrain strategies, as is shown in Figure 3f. ${ }^{[22,26]}$ The structures shown in Figure S9a (Supporting Information) are encapsulated by a $500-\mu \mathrm{m}$-thick layer of silicone (Ecoflex, Smooth-On, Inc.). FEA results (Figure 3f, star markers) indicate that the encapsulation slightly lowers the stretchability for structures in the wrinkling or buckling regimes, but that it has almost no effect on the thick structures $(>45 \mu \mathrm{m})$ in the scissoring regime. Here, the thick metal interconnect is so much stiffer than the soft encapsulation layer that the encapsulation does not alter the deformation characteristics. Figure S9b (Supporting Information) shows a fractal serpentine interconnect, which replaces the straight segments in Figure 1a with curved segments that have a radii of curvature of $0.15 \mathrm{~mm}$. This fractal design, when sufficiently thick (>45 $\mu \mathrm{m})$ to promote the scissoring mode, enhances the elastic stretchability from $96 \%$ to $120 \%$ (Figure 3f, triangle markers). The fractal designs also take advantage of the scissoring thick interconnects placed in multiple directions, as a practical route toward multidirection deformation. Adoption of prestrain (Figure S9c, Supporting Information) can further improve the elastic stretchability. The prestrain should be restricted to values that, upon release, induce deformations within the elastic limit. Prestrain can increase the elastic stretchability of the scissoring structures $(>45 \mu \mathrm{m}$ ) from $96 \%$ to $220 \%$ (Figure 3f, round markers).

Interconnects for Stretchable Arrays of LEDs: This demonstration involves an optimized structure of copper $(45 \mu \mathrm{m}$ thickness; $45 \mu \mathrm{m}$ width) for electrical interconnects between a collection of four LEDs supported by a thin silicone substrate (Ecoflex, $0.5 \mathrm{~mm}$ thickness) (see the Experimental Section for details). Figure 4a shows a functioning LED array in its original geometry and under 245\% applied strain. Stretching beyond this level causes fracture of the substrate but not the interconnects or their bonded interfaces to the LEDs. This level of stretchability is more than 2.5 times larger than that obtained with buckled structures in otherwise similar overall layouts. ${ }^{[33]}$ The system remains functional after 20000 cycles of stretching to $110 \%$, as shown in the top left frame of Figure $4 \mathrm{~b}$. Similar cyclic testing to $120 \%$ leads to fractures at critical locations shown in the bottom and right frames of Figure 4b, consistent with FEA results shown in Figure 4c. This elastic stretchability, i.e. $110 \%$, is three times larger than that previously obtained with wrinkling/buckling designs. ${ }^{[22,34]}$ In addition to superior stretchability, the array also shows excellent electric/thermal performance, due to the large interconnect thickness $(\approx 45 \mu \mathrm{m}$, compared to traditional stretchable circuits of $\approx 300 \mathrm{~nm}$ ). Both experimental and FEA results in Figure 4d and Figure S10 (Supporting Information) indicate that after $40 \mathrm{~min}$ of continuous operation at room temperature $\left(24^{\circ} \mathrm{C}\right)$, the LEDs reach thermal steady-state and maximum temperatures of only $82.5^{\circ} \mathrm{C}$ at the LEDs and $68.3^{\circ} \mathrm{C}$ at the interconnects $(45 \mu \mathrm{m}$ thickness). These values are much lower than those $\left(201.6{ }^{\circ} \mathrm{C}\right.$ at the LEDs and $131.8^{\circ} \mathrm{C}$ at the interconnects) for the wrinkled system $(\approx 300 \mathrm{~nm}$ thickness) under the same operational condition (Figure $4 \mathrm{e}$ ). The thick interconnects have reduced resistances and therefore reduced power consumption by Joule heating; they also have increased effectiveness in heat dissipation.

Interconnects for Stretchable Arrays of Solar Cells: Compact, stretchable sources of power can be important for a range of applications. The thick metal layers with the scissoring design lead to interconnect resistances $(45 \mu \mathrm{m}$ thick, $\approx 0.4 \Omega$ for each wire shown in the structure in Figure S11a, Supporting Information) that are much lower than those of thin, wrinkled structures (e.g., $300 \mathrm{~nm}$ thick, $\approx 60 \Omega$ for each wire). When integrated with solar cells, these low resistances are particularly important because they minimize parasitic power loss. Interconnects for a $2 \times 2$ array of solar cells (MicroLink Devices, dual junction $\mathrm{GaAs} / \mathrm{InGaP}$ ) on a silicone substrate yield a highly stretchable system (Figure 5a, top left frame).

Characterization of the mechanical and electrical performance involves bi-axial stretching while monitoring the current-voltage response under illumination with an AM 1.5 solar simulator. The results, shown in Figure $5 \mathrm{~b}$, indicate stable mechanical characteristics and invariant electrical performance with a short-circuit current of $\approx-3.92 \mathrm{~mA}$ and an open-circuit voltage of $\approx 4.42 \mathrm{~V}$, corresponding to an efficiency of $\approx 14.8 \%$ (computed based on the combined area of the solar cells). The efficiency of the solar cells remains constant for bi-axial stretching up to $110 \%$. For stretching to $120 \%$, the contact pads begin to delaminate (Figure $5 \mathrm{~b}$ red curve in the inset and Figure 5c). This bi-axial elastic stretchability is more than $\approx 2$ times larger than that achieved with buckled interconnects $(60 \%) .{ }^{[35,36]}$ The electroplated copper traces may have slightly different mechanical properties (e.g., yield strength) than those of the laser-milled samples. However, since the designed electroplated interconnects do not fracture during the testing, the effect of the difference in material properties is not discussed here. Although the scissoring mechanism is essentially for $1 \mathrm{D}$ stretching, it is observed here that multiple scissoring interconnects can be oriented in different directions, thereby providing a means to accommodate 2D stretching.

Traces for Stretchable RF Antennas: Radio frequency antennas are critical components of wireless communications and power delivery systems. To minimize resistive losses, the thickness of the metal traces should be significantly larger than the skin depth, which characterizes the penetration depth of electromagnetic waves in the metal. For copper at $1 \mathrm{GHz}$, the skin depth is $\approx 2 \mu \mathrm{m}$. Unlike traditional buckling approaches to stretchable mechanics, which involve thicknesses of a few hundred nanometers, scissoring based schemes allow operation with thick structures.

Radio frequency dipole antennas constructed using the scissoring design consist of a mesh of enclosed metallic unit cells, each of which comprises four 1D interconnect patterns 
a

www.advancedsciencenews.com
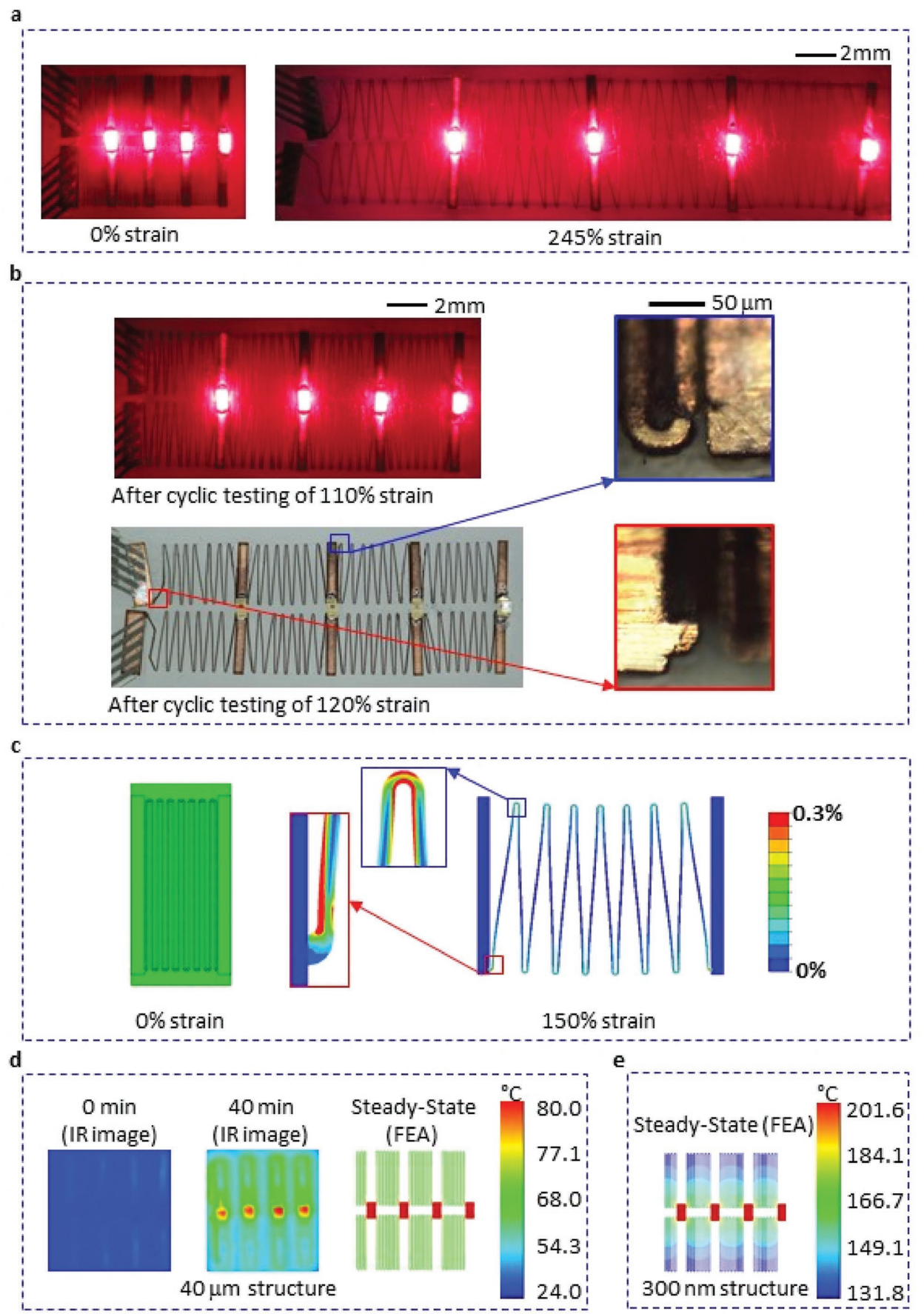

Figure 4. Demonstration of scissoring interconnects in a stretchable array of LEDs. a) Array of LEDs during operation in the original (left) and stretched (right) configurations. b) Array after cyclic testing to strains of $110 \%$ (top) and $120 \%$ (bottom). Fracture locations after cyclic testing to $120 \%$ strain appear in the frames on the right. c) FEA results for the strain distribution in the interconnect at stretching to $150 \%$. d) Steady-state temperature distributions obtained from IR imaging and FEA for interconnects with thicknesses of $40 \mu \mathrm{m}$. e) Steady-state temperature distributions obtained from FEA for interconnects with thicknesses of $300 \mathrm{~nm}$.

(Figure 5d and Figure S12a and S13, Supporting Information). The dimensions of each unit cell (millimeter-scale) are much smaller than the operating wavelength $(30 \mathrm{~cm}$ in free space at $1 \mathrm{GHz}$ ). As such, the mesh of unit cells can be treated as an effective metallic surface, in the form of an electric metamaterial. We consider two different unit cell layouts: one with three serpentine serpentine cycles on each unit cell side (Figure 5d) and one with a single serpentine cycle on each unit cell (Figure S12a, Supporting Information). From mechanical simulations, antennas with the single-serpentine layout can stretch to $32 \%$, while antennas with additional serpentine cycles per cell can stretch to $40 \%$. The stretchability of 


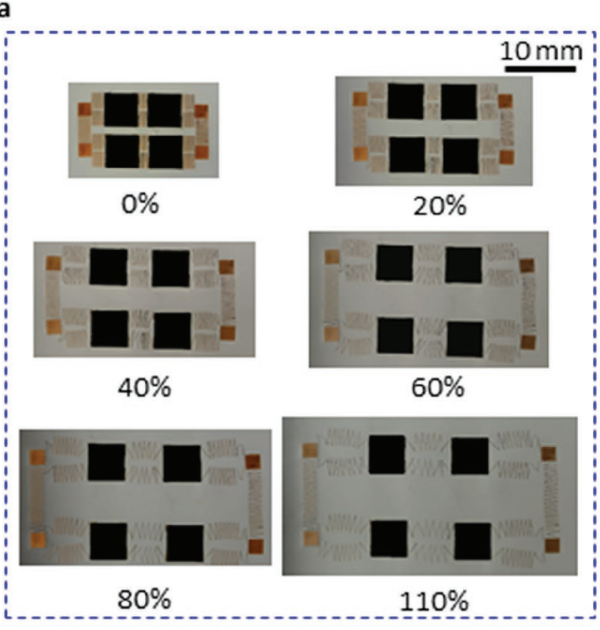

d

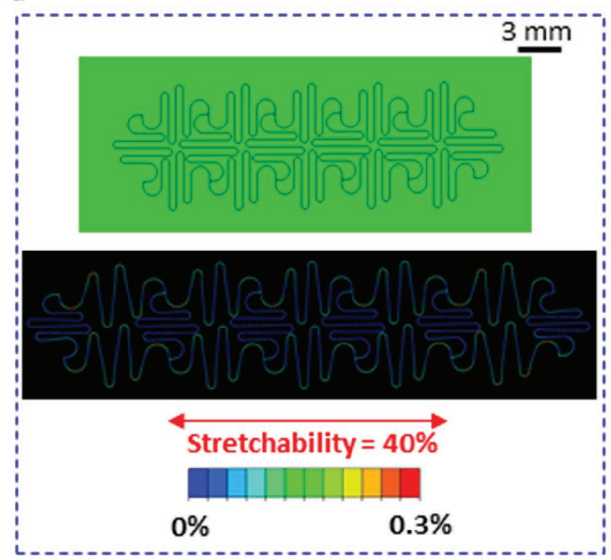

f

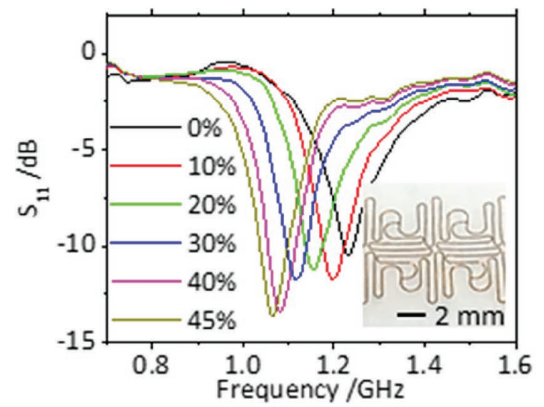

b
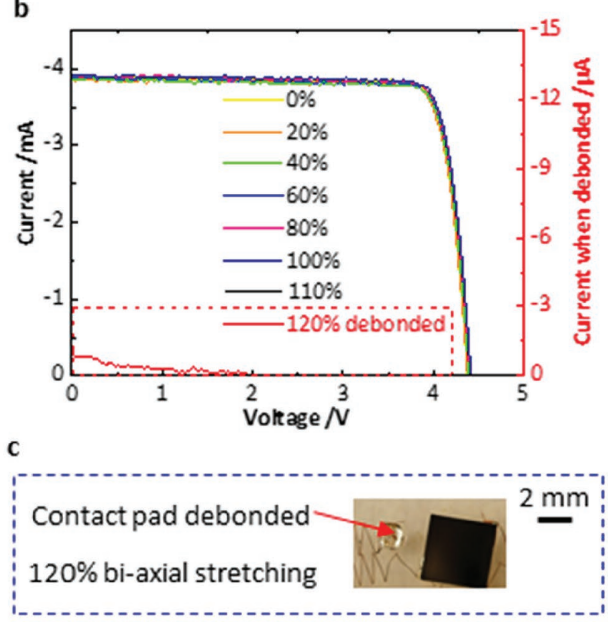

e

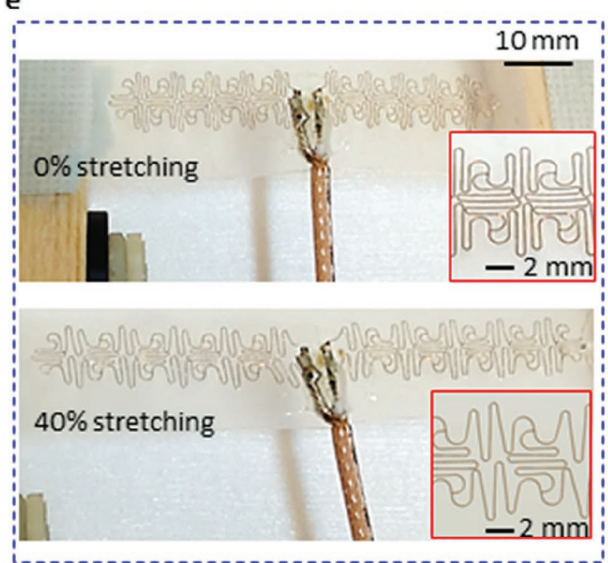

g

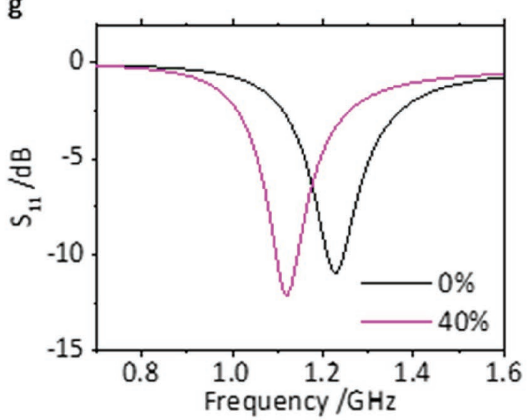

Figure 5. Demonstration of scissoring interconnects in a stretchable array of solar cells and radio frequency dipole antennas. a) Solar cell arrays stretched bi-axially to various levels of strain (0\%, $20 \%, 40 \%, 60 \%, 80 \%$, and $110 \%)$. b) Current-voltage curves that correspond to these levels of stretching. c) Solar cells debonded from the contact pads due to biaxial stretching to $\approx 120 \%$. d) Mechanical simulations of stretched antenna arms comprising unit cells with three serpentine cycles. e) Images of antennas bonded to an elastomer at different levels of strain. SMA cables soldered at the antenna feed points allow probing of the radiofrequency properties. f) Experimentally measured $S_{11}$ return loss spectra for antennas with unit cells consisting of three serpentine cycles, under different strains. g) $S_{11}$ return loss spectra by FEM simulations for antennas with unit cells consisting of three serpentine cycles, under different strains.

these systems is larger than the $30 \%$ stretchability of previously reported designs ${ }^{[37]}$ with $4-\mu \mathrm{m}$-thick copper wires due to the adoption of the scissoring physics.

The use of thick metal $(30 \mu \mathrm{m})$ in these layouts leads to highly stretchable antennas. Testing involves soldering a $50 \mathrm{Ohm}$ SMA cable at the antenna feed point and connecting it to a network analyzer for $\mathrm{S}_{11}$ return loss characterization.
The return loss spectra of the antennas at different levels of mechanical strain appear in Figure 5f. The design with three serpentine cycles has a resonance at $1.24 \mathrm{GHz}$ at $0 \%$ strain, and $1.08 \mathrm{GHz}$ upon $45 \%$ strain, compared to $1.5 \mathrm{GHz}$ at $0 \%$ strain and $1.3 \mathrm{GHz}$ upon $30 \%$ stretching for the design with a single serpentine cycle (Figure S12, Supporting Information). The resonance frequency of a linear dipole antenna scales inversely 
with the length of its arms, due to a combination of changes geometry and associated capacitive coupling. The reduction and narrowing of the $S_{11}$ parameter as the antenna stretches results from enhanced impedance matching between the antenna and $50 \mathrm{Ohm}$ SMA cable upon stretching. Radio frequency FEM simulations (see the Supporting Information for details) also display a strong dependence of antenna resonant frequency on mechanical strain. The antenna with three serpentine cycles in each unit cell resonates at $1.23 \mathrm{GHz}$ at $0 \%$ strain, and $1.12 \mathrm{GHz}$ at $40 \%$ strain (Figure $5 \mathrm{~g}$ ). The antenna consisting of a single serpentine cycle in each unit cell resonates at $1.52 \mathrm{GHz}$ at $0 \%$ strain, and $1.29 \mathrm{GHz}$ at 32\% stretching (Figure S12d, Supporting Information). These theoretical results agree well with the experimental results shown in Figure $5 \mathrm{~d}$ and Figure S12a (Supporting Information). Measurements of the $S_{11}$ parameter of both antennas as a function of mechanical cycling is displayed in Figure S14 (Supporting Information) and showed that as the antenna stretches and relaxes, there are no hysteresis effects and the resonant frequency and line shape are preserved.

The findings presented here demonstrate that scissor-like mechanics represents an important design approach that can complement previously reported schemes in stretchable electronics, where high elastic stretchability, high areal coverages of active devices, and high electric performance can be achieved simultaneously (by high performance, we refer to the low resistances and excellent thermal characteristics associated with thick interconnects.). More generally, systematic studies involving experimental work, FEA and analytical theory reveal three different deformation modes, wrinkling, buckling and scissoring, for serpentine structures of hard materials on soft elastomeric substrates. For otherwise comparable designs, the elastic stretchability in the scissoring regime is much higher than that in other two regimes. Analytical studies of these designs identify key geometric parameters that govern the elastic stretchability and yield optimal values for metallic serpentine interconnects that reach levels of stretchability up to $350 \%$, roughly six times larger than previously reported values $(\approx 60 \%)^{[22]}$ when prestrain is not applied. The scissoring physics depends only on the thickness/width aspect ratio, and the stretchability is reversely proportional to the width. As a result, designs that involve thin interconnects with comparable (small) widths represent optimal options to achieve both large stretchability and large flexibility. Effects of encapsulation, fractal design layouts and prestrain strategy are studied systematically. The scissoring design also provides low electrical resistance and efficient heat dissipation in interconnect structures due to their thick geometries. Demonstrations include interconnects in arrays of high-power LEDs and high performance solar cells. Stretchable radio frequency antenna platforms illustrate the ability to reduce losses from skin-depth effects. The mechanics and technical approaches are distinct from those traditionally exploited in stretchable electronics, thereby providing some important, additional design options and technical capabilities.

\section{Experimental Section}

Fabrication of Thin Metal Structures $(<1 \mu \mathrm{m})$ : Spin-casting yielded a bilayer of poly(methyl methacrylate) (PMMA 495, MicroChem, at speed of 3000 r.p.m. for $30 \mathrm{~s}$, baked on a hotplate at $180^{\circ} \mathrm{C}$ for $2 \mathrm{~min}$ ) and a layer of PI (from poly (pyromellitic dianhydride-co-4,40-oxydianiline) amic acid solution; $\approx 1.5 \mu \mathrm{m}$, Sigma-Aldrich, 4000 r.p.m. for $40 \mathrm{~s}$, baked on a hotplate at $150{ }^{\circ} \mathrm{C}$ for $5 \mathrm{~min}$ and then baked at $250{ }^{\circ} \mathrm{C}$ in vacuum for $1.5 \mathrm{~h}$ ) on a silicon wafer. A layer of copper $(300 \mathrm{~nm}$, or $1.0 \mu \mathrm{m})$ was then deposited by electron beam evaporation onto the PI. Photolithography (AZ 4620) and etching (Type CE-100 copper etchant, Transene Company, at room temperature for $\approx 10 \mathrm{~s}$ ) defined patterns in the metal layer. Next, oxygen plasma etching (180 mTorr, $20 \mathrm{sccm} \mathrm{O}, 200$ Watt for $20 \mathrm{~min}$ ) patterned the $\mathrm{PI}$ in a geometry matched to the metal traces.

Immersion in hot acetone $\left(80^{\circ} \mathrm{C}\right)$ undercut the bottom PMMA layer, thereby allowing the entire structure to be retrieved from the silicon wafer onto the surface of a piece of water-soluble tape (3M). Electron beam evaporation of $\mathrm{Cr}(5 \mathrm{~nm}) / \mathrm{SiO}_{2}(50 \mathrm{~nm})$ formed backside coatings to enhance adhesion to a receiving substrate. Thin silicone slabs (Ecoflex $\approx 0.5 \mathrm{~mm}$ thick, Smooth-On) were prepared by mixing the two constituent components in a 1:1 weight ratio, casting the resulting material into a Petri dish and then partially curing it $(17 \mathrm{~h}$ at room temperature and then $4 \mathrm{~h}$ at $70^{\circ} \mathrm{C}$ ). The surface of the silicone was treated with UV/Ozone for $4 \mathrm{~min}$, and then the interconnect structures were transferred onto this surface with $\mathrm{SiO}_{2}$ facing down. The entire sample was immersed in water for $12 \mathrm{~h}$ to dissolve the water-soluble tape, thereby completing the fabrication processes.

Fabrication of Thick Metal Structures ( $>1 \mu \mathrm{m}$ ): Copper foils (Annealed Alloy 110, Electro Tough Pitch, smooth finish on both sides, All Foils Inc.) were laminated against a flat surface using water soluble tape (3M). A laser milling machine defined traces with designs determined by a CAD file. An infrared (wavelength 790-820 nm) laser with a power of $0.10 \mathrm{~W}$ and three sequential passes along the edges at a speed of $2.0 \mathrm{~mm} \mathrm{~s}^{-1}$ cut vertically through the copper foil (cross-section shown in Figure S15, Supporting Information). Excess material was removed after the laser milling and the desired traces were left on the flat water-soluble tape for ease of handling.

Electroplating of Thick Metal Structures ( $>45 \mu \mathrm{m}$, for the Solar Cell Array Samples): To fabricate the dense interconnecting traces for the solar cell array, accurate patterning was required and electroplating was adopted. Spin-casting defined a layer of poly (methyl methacrylate) (PMMA 495, MicroChem, at speed of 3000 r.p.m. for $30 \mathrm{~s}$, baked on a hotplate at $180{ }^{\circ} \mathrm{C}$ for $\left.2 \mathrm{~min}\right)$ on a silicon wafer. Seed layers of $\mathrm{Ti}$ $(50 \mathrm{~nm}) / \mathrm{Cu}(300 \mathrm{~nm}) / \mathrm{Ti}(100 \mathrm{~nm})$ were then deposited by electron beam evaporation onto the PMMA. Photolithographic exposure (NR2120000P negative photoresist, Futurrex, $1500 \mathrm{rpm}, 10 \mathrm{~s}$ ) and development (RD6 developer, Futurrex, at room temperature for $\approx 8 \mathrm{~min}$ ) defined a $60 \mu \mathrm{m}$ thick mold on top of the metal layer (Figure S11b, Supporting Information), with desired wires and electrodes exposed. The Ti layer was then etched away using reactive ion etching $\left(5 \mathrm{sccm} \mathrm{O}_{2}, 10 \mathrm{sccm}\right.$ $\left.\mathrm{CF}_{4}, 300 \mathrm{mTorr}, 200 \mathrm{~W}, \approx 30 \mathrm{~s}\right)$ to expose the copper seed layer. Next, the wafer was connected to the negative terminal of a power supply and immersed into the electroplating bath (mixed from $900 \mathrm{~mL} \mathrm{H} \mathrm{H}_{2} \mathrm{O}, 225 \mathrm{~g}$ cupric sulfate powder and $22.5 \mathrm{~mL}$ sulfuric acid). A copper sheet was connected to the positive terminal and immersed into the electroplating bath, aligned in parallel to the wafer. Passing a current density of $15 \mathrm{~mA} \mathrm{~cm}^{-2}$ for $3 \mathrm{~h}$ produced a low-resistive copper layer of $45 \mu \mathrm{m}$. The mold was then stripped with acetone and the exposed seed layers were removed by etching.

Immersion in hot acetone $\left(80^{\circ} \mathrm{C}\right)$ undercut the bottom PMMA layer, thereby allowing the entire structure to be retrieved from the silicon wafer onto the surface of a piece of water-soluble tape $(3 \mathrm{M})$. Electron beam evaporation of $\mathrm{Cr}(5 \mathrm{~nm}) / \mathrm{SiO}_{2}(50 \mathrm{~nm})$ formed backside coatings to enhance the adhesion to a receiving substrate. Thin silicone slabs (Ecoflex $\approx 0.5 \mathrm{~mm}$ thick, Smooth-On) were prepared with their surfaces treated with UV/Ozone for $4 \mathrm{~min}$. The metal wires were transferred onto the silicone with $\mathrm{SiO}_{2}$ facing down. The entire sample was then immersed in water for $12 \mathrm{~h}$ to dissolve the water-soluble tape, thereby completing the fabrication processes. (Figure S11c, Supporting Information).

Fabrication of Silicon Structures: (1) Fabrication of thin serpentine Si ribbons $(300 \mathrm{~nm}, 1.5 \mu \mathrm{m}$ and $20 \mu \mathrm{m})$ began with patterning of ribbons 
and anchor structures on silicon-on-insulator wafers. Photolithography and inductive coupled plasma reactive ion etching (STS ICP RIE, STS Mesc Multiplex Advanced Silicon Etcher) of the top Si layers patterned $\mathrm{Si}$ ribbons with the necessary geometries. Immersion in hydrofluoric acid removed the buried oxide layer and isolated the serpentine Si ribbons from the bulk wafer, leaving small anchors that held the Si ribbons in place. The Si ribbons were then released from the anchors with a piece of water-soluble tape and transferred onto a thin silicon slab (Ecoflex $\approx 0.5 \mathrm{~mm}$ thick, Smooth-On). (2) For structures with thicknesses of 50 and $75 \mu \mathrm{m}$, the process began with spin-coating a layer of water-soluble adhesive (poly(vinyl alcohol) (PVA)) on a silicon wafer. Silicon thin films (University Wafers, double-sided cleaned and mirror finished) with thicknesses of 50 or $75 \mu \mathrm{m}$ were then laminated on top of the PVA for ease of handling. Photolithography with a thick photoresist (AZ 4620, $1.2 \mu \mathrm{m}$ ) followed by inductively coupled plasma reactive ion etching (STS-ICPRIE) defined Si structures in the necessary geometries. The resulting structures were then transferred from the wafer to a thin silicon slab (Ecoflex $\approx 0.5 \mathrm{~mm}$ thick, Smooth-On). In the $\mathrm{Si}$ etching processes of (1) and (2), the STS ICPRIE parameters were adjusted according to the various thicknesses of the silicon films. For example, 80 cycles of etching were required to etch through $50 \mu \mathrm{m}$ of $\mathrm{Si}$, where each cycle involved a plasma deposition step and an etching step. Plasma deposition parameters were as follows: step time $4 \mathrm{~s}$, inductively coupled plasma/platen power $600 / 0 \mathrm{~W}, \mathrm{C}_{4} \mathrm{~F}_{8}$ gas flow rate $110 \mathrm{sccm}$, pressure 94 mTorr. Etching parameters were as follows: step time $8 \mathrm{~s}$, $\mathrm{O}_{2} / \mathrm{SF}_{6}$ gas flow rate $13 / 130 \mathrm{sccm}$, pressure: 94 mTorr, etching power $600 / 12 \mathrm{~W}$.

Cyclic and Ultimate Tensile Strength Testing: Cyclic testing was performed with a Fexural Endurance Tester (CK Trade, Korea) at sets of 20000 cycles at $5 \mathrm{~Hz}$. To determine the elastic stretchability of each sample, the strain level was gradually increased until observation of the onset of microcracking. New samples tested under strains slightly lower than this value verified an elastic response, without cracking. Fracture testing was performed with a customized uniaxial stretcher. Optical microscope images allowed measurement of the elongation of each structure and to identify locations of fractures.

\section{Supporting Information}

Supporting Information is available from the Wiley Online Library or from the author.

\section{Acknowledgements}

Y.S. acknowledges the support from Chinese Academy of Sciences via the "Hundred Talent program", the National Science Foundation of China (NSFC, No. 11572323), and the State Key Laboratory of Structural Analysis for Industrial Equipment at Dalian University of Technology (No. GZ1603). X.P. acknowledges the support of NSFC (No. 51365013) and the Natural Science Foundation of Jiangxi Province of China through grant No. 20133ACB21002. J.W.L. and U.P. thank the GRL Program (K20704000003TA050000310) through the NRF funded by the Ministry of Science. S.W. acknowledges the support from the ASME Applied Mechanics Division-Haythornthwaite Foundation Research Initiation Grant, and partial support from the NSFC (Nos. 11272260, 11172022, 11572022, 51075327, and 11302038). Y.H. acknowledges the support from NSF (DMR-1121262, CMMI-1300846, CMMI-1400169, and IIP-1534120). J.A.R. acknowledges supports from the U.S. Department of Energy, Office of Science, Basic Energy Sciences, under Award \# DE-FG02-07ER46741.

Received: September 16, 2016 Revised: October 27, 2016 Published online: December 22, 2016
[1] J. A. Rogers, Nature 2010, 468, 177

[2] D. H. Kim, N. Lu, Y. Huang, J. A. Rogers, MRS Bull. 2012, 37, 226.

[3] T. Someya, T. Sekitani, S. Iba, Y. Kato, H. Kawaguchi, T. Sakurai, Proc. Natl. Acad. Sci. USA 2004, 101, 9966

[4] M. Ramuz, B. C. K. Tee, J. B. H. Tok, Z. Bao, Adv. Mater. 2012, 24, 3223.

[5] J. Lee, J. Wu, M. Shi, J. Yoon, S. I. Park, M. Li, Z. Liu, Y. Huang, J. A. Rogers, Adv. Mater. 2011, 23, 986.

[6] D. J. Lipomi, Z. Bao, Energy Environ. Sci. 2011, 4, 3314.

[7] F. Axisa, P. M. Schmitt, C. Gehin, G. Delhomme, E. McAdams, A. Dittmar, IEEE Trans. Inf. Technol. Biomed. 2005, 9, 325.

[8] S. I. Park, Y. Xiong, R.-H. Kim, P. Elvikis, M. Meitl, D.-H. Kim, J. Wu, J. Yoon, C. J. Yu, Z. Liu, Science 2009, 325, 977.

[9] D. H. Kim, J. H. Ahn, W. M. Choi, H. S. Kim, T. H. Kim, J. Song, Y. Y. Huang, Z. Liu, C. Lu, J. A. Rogers, Science 2008, 320, 507.

[10] M. S. White, M. Kaltenbrunner, E. D. Głowacki, K. Gutnichenko, G. Kettlgruber, I. Graz, S. Aazou, C. Ulbricht, D. A. Egbe, M. C. Miron, Nat. Photonics 2013, 7, 811.

[11] D. H. Kim, N. Lu, R. Ma, Y. S. Kim, R. H. Kim, S. Wang, J. Wu, S. M. Won, H. Tao, A. Islam, Science 2011, 333, 838.

[12] J. Viventi, D. H. Kim, J. D. Moss, Y. S. Kim, J. A. Blanco, N. Annetta, A. Hicks, J. L. Xiao, Y. G. Huang, D. J. Callans, J. A. Rogers, B. Litt, Sci. Transl. Med. 2010, 2, 24ra22.

[13] H. C. Ko, M. P. Stoykovich, J. Song, V. Malyarchuk, W. M. Choi, C. J. Yu, J. B. Geddes III, J. Xiao, S. Wang, Y. Huang, J. A. Rogers, Nature 2008, 454, 748.

[14] Y. M. Song, Y. Xie, V. Malyarchuk, J. Xiao, I. Jung, K. J. Choi, Z. Liu, H. Park, C. Lu, R. H. Kim, Nature 2013, 497, 95.

[15] Y. Zhang, S. Wang, X. Li, J. A. Fan, S. Xu, Y. M. Song, K. J. Choi, W. H. Yeo, W. Lee, S. N. Nazaar, B. Lu, L. Yin, K. C. Hwang, J. A. Rogers, Y. Huang, Adv. Funct. Mater. 2014, 24, 2028.

[16] Y. Su, J. Wu, Z. Fan, K. C. Hwang, J. Song, Y. Huang, J. A. Rogers, J. Mech. Phys. Solids. 2012, 60, 487.

[17] D. H. Kim, J. Song, W. M. Choi, H. S. Kim, R. H. Kim, Z. Liu Y. Y. Huang, K. C. Hwang, Y. W. Zhang, J. A. Rogers, Proc. Natl. Acad. Sci. USA 2008, 105, 18675

[18] C. H. Lee, Y. Ma, K. I. Jang, A. Banks, T. Pan, X. Feng, J. S. Kim, D. Kang, M. S. Raj, B. L. McGrane, B. Morey, X. Wang, R. Ghaffari, Y. Huang, J. A. Rogers, Adv. Funct. Mater. 2015, 25, 3698.

[19] S. Xu, Y. Zhang, L. Jia, K. E. Mathewson, K. I. Jang, J. Kim, H. Fu, X. Huang, P. Chava, R. Wang, S. Bhole, L. Wang, Y. J. Na, Y. Guan, M. Flavin, Z. Han, Y. Huang, J. A. Rogers, Science 2014, 344, 70.

[20] S. Xu, Y. Zhang, J. Cho, J. Lee, X. Huang, L. Jia, J. A. Fan, Y. Su, J. Su, H. Zhang, H. Cheng, B. Lu, C. Yu, C. Chuang, T. i. Kim, T. Song, K. Shigeta, S. Kang, C. Dagdeviren, I. Petrov, P. V. Braun, Y. Huang, U. Paik, J. A. Rogers, Nat. Commun. 2013, 4, 1543.

[21] Y. Su, S. Wang, Y. Huang, H. Luan, W. Dong, J. A. Fan, Q. Yang, J. A. Rogers, Y. Huang, Small 2015, 11, 367.

[22] J. A. Fan, W. H. Yeo, Y. Su, Y. Hattori, W. Lee, S. Y. Jung, Y. Zhang, Z. Liu, H. Cheng, L. Falgout, M. Bajema, T. Coleman, D. Gregoire, R. J. Larsen, Y. Huang, J. A. Rogers, Nat. Mater. 2014, 5, 3266.

[23] G. Lanzara, J. Feng, F. K. Chang, Smart Mater. Struct. 2010, 19 045013.

[24] G Lanzara, N. Salowitz, Z. Q. Guo, F. K. Chang, Adv. Mater. 2010, 22, 4643.

[25] D. S. Gray, J. Tien, C. S. Chen, Adv. Mater. 2004, 16, 393.

[26] D. Y. Khang, H. Q. Jiang, Y. Huang, J. A. Rogers, Science 2006, 311, 208.

[27] H. Jiang, D. Y. Khang, J. Song, Y. Sun, Y. Huang, J. A. Rogers, Proc. Natl. Acad. Sci. USA 2007, 104, 15607.

[28] S. Yang, E. Ng, N. Lu, Extreme Mech. Lett. 2015, 2, 37.

[29] This maximum exists because the interconnect is made of copper and PI. For a single-layered material there is no maximum.

[30] A. Gjelsvik, The Theory of Thin Walled Bars, Wiley, New York 1981. 
[31] W. F. Riley, L. D. Sturges, D. H. Morris, Mechanics of Materials, Jon Wiley \& Sons, New York 1999.

[32] P. Bartolo, J. Vasco, B. Silva, C. Galo, Assem. Autom. 2006, 26, 227.

[33] R. H. Kim, M. H. Bae, D. G. Kim, H. Cheng, B. H. Kim, D. H. Kim, M. Li, J. Wu, F. Du, H. S. Kim, Nano Lett. 2011, 11, 3881.

[34] X. Ho, C. K. Cheng, R. L. S. Tan, J. Wei, J. Mater. Res. 2015, 30, 2271.
[35] J. Lee, J. Wu, J. H. Ryu, Z. Liu, M. Meitl, Y. W. Zhang, Y. Huang, J. A. Rogers, Small 2012, 8, 1851

[36] S. Choi, H. Lee, R. Ghaffari, T. Hyeon, D. H. Kim, Adv. Mater. 2016, 28, 4203.

[37] A. M. Hussain, F. A. Ghaffar, S. I. Park, J. A. Rogers, A. Shamim, M. M. Hussain, Adv. Funct. Mater. 2015, 25, 6565. 